\title{
A voltammetric tool for the evaluation of propolis antioxidant activity
}

\author{
Soraia I. Falcão ${ }^{1,2} \cdot$ Andreia Tomás $^{1} \cdot$ Cristina Freire $^{2} \cdot$ Miguel Vilas-Boas $^{1}$
}

Received: 24 September 2015 / Revised: 10 December 2015 / Accepted: 23 January 2016 / Published online: 8 February 2016

(C) Springer-Verlag Berlin Heidelberg 2016

\begin{abstract}
Propolis (bee glue) is a sticky dark-colored material that honeybees collected from parts of plants, buds and exudates, and used as construction and defense material in the hive. This resinous substance is a rich source of phenolic compounds, and its chemical composition is strongly influenced by its phytogeographic origin. This research outlines the evaluation of the redox profile and the quantification of the total antioxidant capacity in Portuguese propolis from different origins and potential floral sources Populus $x$ canadensis Moench and Cistus ladanifer $\mathrm{L}$ through cyclic voltammetry and differential pulse voltammetry. Although several oxidation processes were showed by propolis samples, which were dependent of the resin origin, common oxidation potentials within the majority of samples of a region were detected, suggesting an analogous chemical composition in respect of electroactive species. Based on the quantification of the total electroactive antioxidant power, it was found that propolis with higher antioxidant activity was from coast center, followed by north $\sim$ Azores Archipelago $>$ central interior $>$ Madeira Island $>$ south.
\end{abstract}

Keywords Propolis · Electrochemistry $\cdot$ Cyclic voltammetry $\cdot$ Differential pulse voltammetry $\cdot$ Antioxidant capacity

Miguel Vilas-Boas

mvboas@ipb.pt

1 CIMO, Escola Superior Agrária, Instituto Politécnico de Bragança, Campus de Sta. Apolónia, Apartado 1172, 5300-253 Bragança, Portugal

2 REQUIMTE, Departamento de Química e Bioquímica, Faculdade de Ciências, Universidade do Porto, Rua do Campo Alegre, s/n, 4169-007 Porto, Portugal

\section{Introduction}

Natural antioxidants are primarily polyphenols that may occur in many food products of vegetable origin, comprising an extensive and complex group of secondary metabolites that are synthesized during plant growth and as response to stress. They present diverse properties as they can act as reducing agents (free radical terminators), metal chelators and singlet oxygen quenchers. Mainly, they are constituted from phenolic acids and flavonoids, which have a common structure comprising an aromatic ring with one or more hydroxyl substituents. They play an important role in plants, food and humans, presenting many biological properties, including cardio-protective, anti-inflammatory, anti-allergenic, anti-carcinogenic and antimicrobial $[1,2]$. These properties are attributed to their powerful antioxidant and anti-radical activity, which is related to the redox properties of phenolic compounds [3].

Bee products, especially propolis, proved to be a major source of phenolic compounds [4]. More than 300 substances were identified in propolis including flavonoid glycosides and aglycones, phenolic acids and their esters, phenolic aldehydes, alcohols and ketones, sesquiterpenes, quinones, coumarins, steroids, aminoacids and inorganic compounds [5]. The complex chemical composition of propolis is highly variable, depending strongly on the phytogeographic characteristics around the hive. In Europe, North America and other temperate regions, the predominance Populus species makes them the main source of the bee glue $[5,6]$. Generally, temperate propolis is composed by flavonoids without B-ring substituents, such as pinocembrin, pinobanksin, galangin and chrysin and phenylpropanoid acids and their esters (e.g., caffeic acid, caffeic acid phenethyl ester) [6, 7]. In the bee glue, 
the phenolic fraction is responsible for most the biological and health-enhancing properties like the antioxidant activity [8]. Of late, several protocols have been proposed for measuring the antioxidant properties in different propolis $[9,10]$. Antioxidant capacity assays can be roughly divided into two categories, depending on the chemical reaction involved: hydrogen atom transfer (HAT) and single electron transfer (SET) reaction-based assays. HAT-based assays monitor competitive reaction kinetics [i.e., oxygen radical absorbance capacity assay (ORAC) and the total radical-trapping antioxidant parameter assay (TRAP)]. The SET-based assays involve one redox reaction with the oxidant [i.e., Trolox equivalent antioxidant capacity assay (TEAC), ferric ion reducing antioxidant parameter assay (FRAP), DPPH-based assay, copper (II) reduction capacity and total phenolics assay by Folin-Ciocalteu (FC)] [11]. Most of these methods are based on spectrophotometric techniques and present several drawbacks like the relatively higher cost due to the need of reactive species. Moreover, many antioxidant assays suffer from interference when working with colorful or turbid samples or when interfering compounds (i.e., vitamin C, sugars) are abundantly present in the samples [12].

Taking advantage of the electrochemical activity present by the phenolic compounds, resulting from the electronic delocalization on the aromatic nucleus, they can be easily oxidized at inert electrodes and therefore be subject of study by electrochemical methods. Its overall reducing power can be evaluated within food and biological samples without the use of specific reagents [11]. An important relationship between electrochemical behavior and antioxidant power was already established: A low oxidation potential is associated with a high antioxidant power [13]. On the other hand, the intensity of the anodic current is related to the total content of the reducing species present in the sample [14]. Some authors have already applied electrochemical approaches regarding to propolis analysis, in particular evaluation of the antioxidant activity by amperometric flow injection analysis [15], cyclic voltammetry [16] and polarography [17]. Also, the redox properties of specific isolated compounds from propolis were investigated by cyclic voltammetry [18]. To our knowledge, the use of differential pulse voltammetry, applied in the evaluation of other food $[19,20]$ and non-food matrix [21], has never been explored in propolis.

The aim of this work was to establish a rapid, easy and low-cost tool, for the evaluation of the antioxidant capacity in propolis from different origins, by using electrochemical methods like cyclic voltammetry and differential pulse voltammetry. In addition, this new approach allowed the discrimination of samples in accordance with the potential floral sources by analyzing buds exudates and surface material present on the leaves and stems of Populus $x$ canadensis
Moench (poplar), male and female specimens, and Cistus ladanifer L. (rockrose).

\section{Materials and methods}

\section{Chemicals}

Standard compounds such as galangin and caffeic acid were purchased from Sigma Chemical Co (St. Louis, MO, USA). Pinocembrin was from Latoxan (Valence, France). Tetrabutylammonium perchlorate (TBAP), electrochemical grade, was from Fluka (Sigma Chemical Co., St. Louis, MO, USA) and kept at $30^{\circ} \mathrm{C}$ before use. All other chemicals were obtained from Panreac (Barcelona, Spain). Water was treated in a Milli-Q water purification system (Topway Global Inc., Houston, TX, USA).

\section{Samples origin and preparation}

The study was performed on propolis and plants available in the hive neighborhood and reported [5, 22] as propolis floral sources. Thirty-nine propolis samples were collected from six different geographic regions in Portugal [7]: continental north (N1-6, Bragança; N7, Miranda do Douro; N8, Mirandela; N9-10, Chaves; N11, Montalegre; N12-13, Boticas; N14, Barcelos); central interior (CI1, Guarda; CI3, Fundão; CI4, Nisa); central coast (CC1, Figueira da Foz; CC2, Leiria; CC3, Coruche; CC4, Ramada); south (S1-3, Aljezur; S4, Moncarapacho); Azores Archipelago (A1, Terceira Island; A2-11, S. Miguel Island) and Madeira Island (M1-3, Funchal, Madeira Island). All the samples were obtained between 2007 and 2009 after the honey harvesting season (July/September), by conventional scraping or through plastic screens.

For propolis floral sources, the study was performed on plant samples available in the hive neighborhood that were reported as propolis floral sources [5]. Leaf buds from Populus $x$ canadensis Moench (an hybrid species of Populus) male (PM) and female (PF) and Cistus ladanifer L. (C) branches, in the earlier floral stage, were collected from a minimum of five wild-growing plants in the Bragança region, northeast Portugal, in the spring of 2009. The voucher specimens are deposited at the herbarium of Escola Superior Agrária of Instituto Politécnico de Bragança with the reference number BRESA 5174, BRESA 5355 and BRESA 5356 for C, PF and PM, respectively. All plant material samples were stored at $-20{ }^{\circ} \mathrm{C}$ prior to phenolic extraction.

The phenolic extraction of propolis and of the plant sources was made according to the work previously described [7]. Prior to the extraction, $1 \mathrm{~g}$ of powdered sample was homogenized and mixed with $10 \mathrm{~mL}$ of $80 \%$ 
of ethanol/water and kept at $70{ }^{\circ} \mathrm{C}$ for $1 \mathrm{~h}$. The resulting mixtures were filtered, and the residues were re-extracted in the same conditions. After the second extraction, the filtrates were combined, concentrated and freeze-dried. The extracts were used as obtained, without further purification.

\section{Electrochemical characterization}

\section{Instrumentation}

Cyclic voltammetry (CV) and differential pulse voltammetry (DPV) measurements were performed on an Autolab PGSTAT 302 potentiostat/galvanostat using a closed standard three-electrode cell. A glassy carbon $\left(\phi=0.314 \mathrm{~cm}^{2}\right)$ was used as the working electrode and a Pt foil as the counter electrode. All potentials refer to an $\mathrm{Ag} / \mathrm{AgCl} 3$ moldm $^{-3}$ $\mathrm{KCl}$ reference electrode. Prior to use, the working electrode was polished in an aqueous suspension of $0.3 \mu \mathrm{m}$ alumina (Beuhler) on a Master-Tex (Beuhler) polishing pad, than rinsed with water. Subsequently, in a chemical treatment, the electrode was sonicated in $\mathrm{HCl} 6$ moldm $^{-3}$ for $1 \mathrm{~min}$ and then in methanol. This cleaning procedure was applied always before any electrochemical measurements.

\section{Procedure}

Propolis plants' ethanolic extracts and standard compounds were studied in ethanol/Britton-Robinson buffer/ TBAP (78:20:2) at $\mathrm{pH} 7$ because the oxidation of polyphenols at neutral $\mathrm{pH}$ mimics physiological conditions [23]. The extract solutions were prepared in a concentration of $1 \mathrm{mg} \mathrm{mL}{ }^{-1}$. All solutions were analyzed immediately after preparation, and the electrochemical response was recorded after the immersion of the glassy carbon electrode, to minimize adsorption of species onto the electrode surface prior to the run. The working electrode surface state was previously checked with the analysis of the ethanol/Britton-Robinson buffer/TBAP (78:20:2) solution alone. Every experiment was performed in triplicate, and the results are shown as an average.

The characterization of the electrochemical response process was achieved using cyclic voltammetry between 0 and $1.5 \mathrm{~V}$, at $0.1 \mathrm{Vs}^{-1}$ as scan rate. For the evaluation of antioxidant capability, the operating conditions for DPV were set at $0.06 \mathrm{~V}$ pulse amplitude and $0.030 \mathrm{Vs}^{-1}$ as scan rate, between -0.25 and $1.5 \mathrm{~V}$.

\section{Quantification of the total electrochemical antioxidant power}

The quantification procedure was performed at $\mathrm{pH}=7$ considering the intensity of the electrochemical response. For that, a calibration curve $(y=75.4590-0.2569 \times$;
$\left.R^{2}=0.997\right)$ was prepared by plotting the concentration of a standards mixture representative of the phenolic composition of propolis [caffeic acid: galangin: pinocembrin (1:1:1)] against the current density of the respective DPV signals, using several solutions in the range 0.01-0.10 $\mathrm{mgmL}^{-1}$. The analytical signal of the samples (current intensity) was measured between peak maxima and the baseline, defined as the tangent between the lowest and the highest potential valley. This current signal was expressed in terms of equivalents of standard solution [caffeic acid: galangin: pinocembrin (1:1:1)] per gram of propolis extract $\left(\mathrm{mgg}^{-1}\right)$. The sum of the values calculated at peak maxima for each electrochemical process was used to express the total electrochemical antioxidant power (TEAP) of propolis samples.

\section{Results and discussion}

Two electrochemical techniques were applied in the study of the propolis ethanolic extracts from different Portuguese regions: The cyclic voltammetry was used for the evaluation of the redox profile of the samples, while the differential pulse voltammetry allowed the quantification of the electroactive species present in the different ethanolic extracts. The electrochemical response of two potential floral sources of propolis was also analyzed in an attempt to establish the plant origin of the samples.

\section{Cyclic voltammetry}

The electrochemical response for the extracts obtained with $\mathrm{CV}$ at $\mathrm{pH} 7$ exhibited a similar voltammetric profile, Fig. 1 (left side), with an irreversible oxidation process characterized by a broad intense anodic wave.

This wave broadening may be due to the electron removal from a mixture of components, within the sample, with the same ability for oxidation, rather than to just an individual component. The location where this anodic process appears in the potential window and the observation of other less intense peaks suggest the existence of samples with different chemical compositions, Table 1.

For the majority of the samples, the higher intensity anodic wave was observed around $0.5-0.7 \mathrm{~V}$, with the exception of samples A10 and A11 from Azores Archipelago and M2 from Madeira Island which reveal oxidation processes at lower potentials, $0.3-0.4 \mathrm{~V}$. This could be due to the presence of more oxidizable species in these extracts and so a higher reducing power [24, 25]. Some samples reveal one or two additional oxidation processes at more positive potentials $(0.8-1.1 \mathrm{~V})$. This behavior was found in some north samples (N10-N14), center interior (CI1-CI4), south (S4) and Madeira Island (M2-3). The appearance of 
Fig. 1 Electrochemical responses for $1 \mathrm{mg} \mathrm{mL}^{-1}$ propolis extracts of sample in EtOH/Britton-Robinson buffer/ TBAP (78:20:2) solution at $\mathrm{pH}$ 7 , obtained with a glassy carbon electrode: (left) cyclic voltammogram at $0.1 \mathrm{~V} \mathrm{~s}^{-1}$ between -0.25 and $1.5 \mathrm{~V}$. a N8, b N11, c A10, d M2 and e blank solution (EtOH/Britton-Robinson buffer/TBAP (78:20:2) solution at $\mathrm{pH} 7)$; (right) differential pulse voltammograms obtained with $0.06 \mathrm{~V}$ pulse amplitude at $0.03 \mathrm{~V} \mathrm{~s}^{-1}$, between -0.25 and 1.5 V. f N8, g N11, h A10, i M2 and $\mathbf{j}$ blank solution (EtOH/ Britton-Robinson buffer/TBAP (78:20:2) solution at $\mathrm{pH} 7$ )

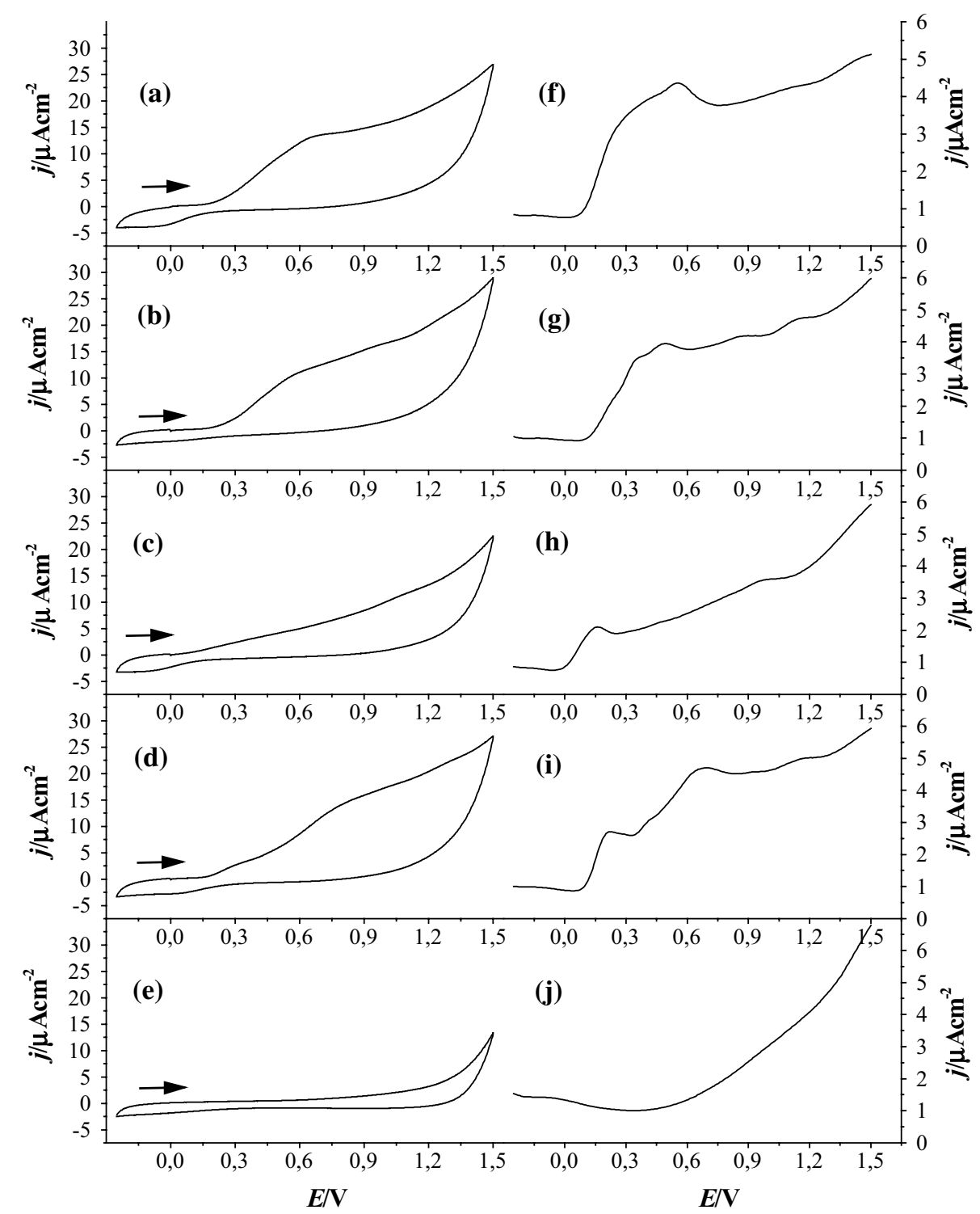

oxidation processes at high potentials can have a limited contribution to the antioxidant activity, since the substances that should be "protected" may have a higher ability to be oxidized before the antioxidant species.

The voltammogram for Cistus ladanifer and Populus $x$ canadensis extracts revealed only one broad anodic irreversible process with similar peak potential for both poplar male and female specimens, around $0.62 \mathrm{~V}$ and slightly higher than that observed for rockrose, Table 1, which was common to the majority of the samples.

\section{Differential pulse voltammetry}

Although the cyclic voltammetry appears in the literature as an electrochemical method used when analyzing antioxidants $[14,25]$, the capacity current associated with the electrochemical system reduces the ability to detect low intense oxidation processes. In the present study, we explore the use of differential pulse voltammetry (DPV) to evaluate the antioxidant capacity rather than CV. DPV is a more selective and sensitive technique, because the measurement of the current signal occurs before and after the potential pulse application allowing the discrimination of effects, like absorption on the electrode by organic substances. Those effects are approximately constants in a limited potential interval, enabling an easier interpretation of the results with better resolved peaks and overcoming the difficulties of accessing a correct baseline common in the cyclic voltammogram, in spite of the higher current density observed [26].

The differential pulse voltammograms of representative propolis samples are showed in Fig. 1 (right side). Through this technique, the number of potential peaks observed is much higher than the ones found by cyclic voltammetry 
Table 1 Peak potentials obtained, at $\mathrm{pH} 7$, for the electrochemical oxidation processes of propolis and floral sources extracts (mean $\pm \mathrm{SD}$, $n=3$ )

\begin{tabular}{|c|c|c|c|c|c|c|c|c|c|c|}
\hline \multirow[t]{2}{*}{ Sample } & \multicolumn{4}{|c|}{ Cyclic voltammetry (V) } & \multicolumn{6}{|c|}{ Differential pulse voltammetry $(\mathrm{V})$} \\
\hline & Ep2 & Ep3 & Ep4 & Ep1 & Ep2 & Ep3 & Ep4 & Ep5 & Ep6 & Ep7 \\
\hline N1 & $0.65 \pm 0.00$ & - & - & - & $0.25 \pm 0.00$ & - & $0.55 \pm 0.00$ & - & - & - \\
\hline $\mathrm{N} 2$ & $0.65 \pm 0.01$ & - & - & - & $0.25 \pm 0.00$ & - & $0.57 \pm 0.00$ & - & - & - \\
\hline N3 & $0.66 \pm 0.06$ & - & - & - & $0.25 \pm 0.00$ & - & $0.57 \pm 0.00$ & - & - & - \\
\hline N4 & $0.67 \pm 0.02$ & - & - & - & $0.23 \pm 0.00$ & - & $0.59 \pm 0.00$ & - & - & - \\
\hline N5 & $0.65 \pm 0.01$ & - & - & - & $0.23 \pm 0.00$ & - & $0.57 \pm 0.00$ & - & - & - \\
\hline N6 & $0.63 \pm 0.00$ & - & - & - & $0.24 \pm 0.00$ & - & $0.56 \pm 0.00$ & - & - & - \\
\hline N7 & $0.64 \pm 0.01$ & - & - & - & $0.24 \pm 0.00$ & $0.42 \pm 0.00$ & $0.56 \pm 0.01$ & - & - & - \\
\hline N8 & $0.63 \pm 0.00$ & - & - & - & $0.28 \pm 0.00$ & - & $0.55 \pm 0.00$ & - & - & $1.10 \pm 0.02$ \\
\hline N9 & $0.60 \pm 0.01$ & - & - & - & $0.23 \pm 0.00$ & - & $0.51 \pm 0.00$ & - & - & $1.06 \pm 0.01$ \\
\hline N10 & $0.58 \pm 0.00$ & - & $1.13 \pm 0.00$ & - & $0.21 \pm 0.00$ & - & $0.51 \pm 0.00$ & - & - & $1.02 \pm 0.01$ \\
\hline N11 & $0.60 \pm 0.02$ & $0.96 \pm 0.00$ & - & - & $0.23 \pm 0.00$ & $0.35 \pm 0.00$ & $0.49 \pm 0.00$ & - & $0.86 \pm 0.00$ & $1.14 \pm 0.00$ \\
\hline N12 & $0.57 \pm 0.01$ & $0.93 \pm 0.00$ & - & - & $0.23 \pm 0.00$ & $0.33 \pm 0.00$ & $0.47 \pm 0.01$ & - & $0.85 \pm 0.00$ & $1.10 \pm 0.01$ \\
\hline N13 & $0.49 \pm 0.01$ & $0.86 \pm 0.01$ & $1.14 \pm 0.00$ & - & $0.20 \pm 0.00$ & $0.29 \pm 0.00$ & $0.41 \pm 0.01$ & - & $0.80 \pm 0.00$ & $1.04 \pm 0.00$ \\
\hline N14 & $0.57 \pm 0.04$ & - & $1.11 \pm 0.00$ & - & $0.20 \pm 0.00$ & $0.37 \pm 0.00$ & $0.51 \pm 0.00$ & - & - & $1.01 \pm 0.00$ \\
\hline CI1 & $0.69 \pm 0.00$ & $0.94 \pm 0.00$ & $1.18 \pm 0.00$ & - & $0.23 \pm 0.00$ & $0.33 \pm 0.00$ & $0.48 \pm 0.00$ & $0.65 \pm 0.00$ & $0.85 \pm 0.00$ & $1.09 \pm 0.01$ \\
\hline $\mathrm{CI} 3$ & $0.57 \pm 0.00$ & $0.84 \pm 0.01$ & $1.15 \pm 0.00$ & - & $0.23 \pm 0.00$ & $0.31 \pm 0.00$ & $0.50 \pm 0.00$ & - & $0.77 \pm 0.01$ & $1.04 \pm 0.00$ \\
\hline CI4 & $0.61 \pm 0.02$ & $0.88 \pm 0.00$ & - & - & $0.23 \pm 0.00$ & $0.41 \pm 0.02$ & $0.52 \pm 0.01$ & - & $0.79 \pm 0.00$ & $1.05 \pm 0.00$ \\
\hline $\mathrm{CC} 1$ & $0.68 \pm 0.01$ & - & - & - & $0.25 \pm 0.00$ & - & $0.57 \pm 0.00$ & - & - & - \\
\hline $\mathrm{CC} 2$ & $0.72 \pm 0.00$ & - & - & - & $0.22 \pm 0.01$ & - & $0.57 \pm 0.00$ & - & $0.82 \pm 0.00$ & - \\
\hline $\mathrm{CC} 3$ & $0.60 \pm 0.01$ & - & - & - & $0.22 \pm 0.00$ & - & $0.52 \pm 0.00$ & - & - & $1.02 \pm 0.01$ \\
\hline $\mathrm{CC} 4$ & $0.64 \pm 0.00$ & - & - & - & $0.26 \pm 0.00$ & - & $0.56 \pm 0.02$ & - & - & $1.06 \pm 0.04$ \\
\hline S1 & $0.70 \pm 0.02$ & - & - & - & $0.23 \pm 0.00$ & - & $0.58 \pm 0.00$ & - & - & $1.11 \pm 0.02$ \\
\hline S2 & $0.59 \pm 0.00$ & - & - & - & $0.23 \pm 0.00$ & - & $0.50 \pm 0.00$ & - & $0.79 \pm 0.00$ & $1.01 \pm 0.00$ \\
\hline S3 & $0.58 \pm 0.01$ & - & - & - & $0.25 \pm 0.00$ & - & $0.52 \pm 0.00$ & - & $0.80 \pm 0.00$ & $1.03 \pm 0.00$ \\
\hline S4 & $0.57 \pm 0.00$ & $0.84 \pm 0.01$ & - & - & $0.20 \pm 0.01$ & - & $0.47 \pm 0.00$ & - & $0.77 \pm 0.01$ & $0.97 \pm 0.00$ \\
\hline A1 & $0.67 \pm 0.01$ & - & - & $0.14 \pm 0.00$ & $0.24 \pm 0.01$ & - & $0.55 \pm 0.00$ & - & - & $1.07 \pm 0.04$ \\
\hline $\mathrm{A} 2$ & $0.74 \pm 0.00$ & - & - & - & $0.23 \pm 0.00$ & - & $0.58 \pm 0.00$ & - & - & - \\
\hline A3 & $0.77 \pm 0.00$ & - & - & - & $0.23 \pm 0.00$ & - & $0.56 \pm 0.00$ & - & - & - \\
\hline A4 & $0.67 \pm 0.06$ & - & - & $0.15 \pm 0.00$ & $0.24 \pm 0.00$ & - & $0.59 \pm 0.00$ & - & - & - \\
\hline A5 & $0.66 \pm 0.02$ & - & - & - & $0.23 \pm 0.01$ & - & $0.55 \pm 0.00$ & - & - & - \\
\hline A6 & $0.72 \pm 0.00$ & - & - & - & $0.26 \pm 0.00$ & - & $0.59 \pm 0.00$ & - & - & - \\
\hline A7 & $0.71 \pm 0.02$ & - & - & $0.16 \pm 0.00$ & - & - & $0.55 \pm 0.00$ & - & - & - \\
\hline A8 & $0.65 \pm 0.00$ & - & - & $0.16 \pm 0.00$ & $0.26 \pm 0.00$ & - & $0.55 \pm 0.00$ & - & & $0.90 \pm 0.00-$ \\
\hline A9 & $0.68 \pm 0.00$ & - & - & - & $0.26 \pm 0.00$ & - & $0.57 \pm 0.00$ & - & - & $1.10 \pm 0.00$ \\
\hline A10 & $0.39 \pm 0.01$ & - & - & $0.14 \pm 0.00$ & - & - & - & - & - & $0.96 \pm 0.01$ \\
\hline A11 & $0.43 \pm 0.02$ & - & - & $0.19 \pm 0.00$ & & - & - & - & - & $1.02 \pm 0.00$ \\
\hline M1 & $0.52 \pm 0.01$ & - & - & - & $0.21 \pm 0.00$ & $0.44 \pm 0.01$ & - & - & $0.74 \pm 0.00$ & $1.02 \pm 0.00$ \\
\hline M2 & $0.31 \pm 0.01$ & $0.79 \pm 0.01$ & - & - & $0.21 \pm 0.01$ & $0.41 \pm 0.00$ & - & $0.64 \pm 0.02$ & - & $1.15 \pm 0.01$ \\
\hline M3 & $0.59 \pm 0.00$ & $0.81 \pm 0.02$ & - & - & - & - & $0.53 \pm 0.01$ & - & $0.75 \pm 0.00$ & - \\
\hline PM & $0.63 \pm 0.03$ & - & - & - & $0.25 \pm 0.00$ & - & $0.55 \pm 0.00$ & - & - & $1.11 \pm 0.02$ \\
\hline $\mathrm{PF}$ & $0.62 \pm 0.01$ & - & - & - & $0.24 \pm 0.00$ & - & $0.52 \pm 0.00$ & - & - & $1.09 \pm 0.01$ \\
\hline $\mathrm{C}$ & $0.56 \pm 0.01$ & - & - & - & $0.17 \pm 0.00$ & $0.33 \pm 0.00$ & - & - & $0.80 \pm 0.00$ & $1.07 \pm 0.00$ \\
\hline
\end{tabular}

and ranges between one in the sample A10 from Azores and the more complex electrochemical profile of sample CI1 from central interior, with six distinct anodic processes,
Table 1. However, some of those oxidation processes had only a low current density and were seen as small inflections in the voltammogram. 
Generally, the propolis samples showed two common oxidation processes around 0.2 and $0.6 \mathrm{~V}$, Table 1, Fig. 1f. Beside those, some propolis samples from Azores Archipelago, namely A1, A4, A7, A8 and A10, presented a lower peak potential around $0.15 \mathrm{~V}$, well observed in sample A10 due to the inexistence, in this sample, of the oxidation process at $0.2 \mathrm{~V}$, Fig. 1h. Despite the less positive potential, there was no correspondence with a higher reducing power [27] as found in other works [19], maybe due to the low intensity of these peaks which could limit the contribution of the oxidation process in the overall activity of the extracts. Most of the samples also present an oxidation peak above $1 \mathrm{~V}$ which was also found in the extract of poplar. Within the common peak potentials, the samples from the north, coast center and Azores Archipelago presented the highest current densities (data not showed), meaning a higher content in oxidizable compounds. This observation is in agreement with the higher content in total phenolics and flavonoids found previously for these samples by spectrophotometric methods [27].

The chemical composition of the propolis is dependent on the floral diversity around the hive, and although the electrochemical response obtained in this study does not reflect the individual phenolic components of propolis, the distinct electrochemical profiles observed in some samples must reflect the different plant sources available to the bees for the resin production. Populus $x$ canadensis male (PM) and female (PF) extracts presented the common oxidation processes at 0.2 and $0.6 \mathrm{~V}$, which is in agreement with the expectable contribution of this plant to the Portuguese propolis as described in the previous works [28], Table 1. For the Cistus ladanifer (C) resin, other oxidation processes were detected at $0.35,0.85$ and $1.05 \mathrm{~V}$. These peaks were also found in propolis samples N11-N13 from the north, CI1-CI4 from central interior and M1 from Madeira Island, while samples S2-S4 from the south presented the last two potentials. Once again, these results are in agreement with the phenolic profile described previously [28], being Cistus resins a potential contributor for the propolis from these regions.

To describe the antioxidant capacity of each propolis sample, the electrochemical current density was quantified in comparison with the electrochemical signal of a standard mixture solution of caffeic acid: galangin: pinocembrin $(1: 1: 1)$, which mimics this complex matrix constituted by different phenolic groups. The electrochemical response of the standard mixture increases in current density with the concentration of the phenolic compounds, as can be observed in the voltammograms of Fig. 2a, leading to a linear correlation between the two parameters, in the range of 0.01-0.06 mg mL $\mathrm{mL}^{-1}$, Fig. 2 b.

Table 2 shows the "electrochemical antioxidant power" (EAP) expressed as equivalents of caffeic acid: galangin:
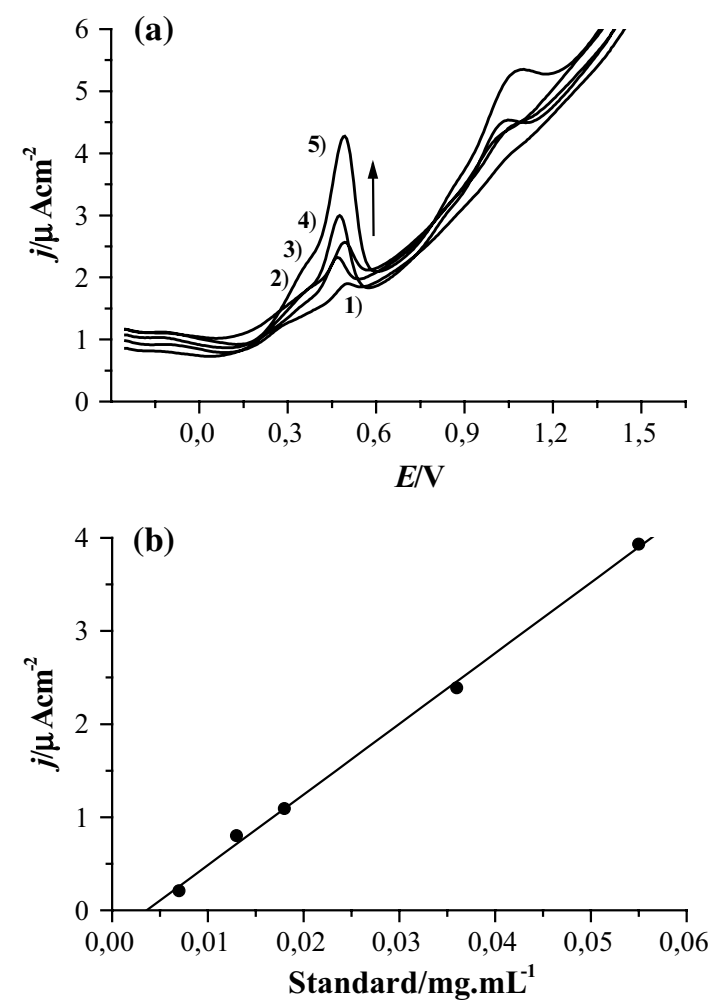

Fig. 2 a Differential pulse voltammograms obtained for the mixture caffeic acid: galangin: pinocembrin $(1: 1: 1)$ used as standard in EtOH/ Britton-Robinson buffer/TBAP (78:20:2) solutions at $\mathrm{pH} 7$, with glassy carbon electrode between -0.25 and $1.5 \mathrm{~V}$, with $0.06 \mathrm{~V}$ pulse amplitude at $0.03 \mathrm{~V} \mathrm{~s}^{-1}$; 1) $0.007 \mathrm{mg} \mathrm{mL}^{-1}$, 2) $0.013 \mathrm{mg} \mathrm{mL}^{-1}$, 3) $0.018 \mathrm{mg} \mathrm{mL}^{-1}$, 4) $0.036 \mathrm{mg} \mathrm{mL}^{-1}$, 5) $0.055 \mathrm{mg} \mathrm{mL}^{-1}$. b Dependence of the oxidation current density, obtained by DPV, with the concentration of the standard mixture

pinocembrin (1:1:1) and calculated for each resolved oxidation peak together with the total electrochemical antioxidant power (TEAP), which resulted from the sum of the different EAP for each sample [19-21]. The "electrochemical antioxidant power" will define the amount of phenolic compounds oxidized at a certain potential, while TEAP will give a general evaluation of the total phenolic composition.

It is clear from the table that the oxidation processes with higher contribution for the TEAP are those with lower potentials. Overall, the highest values of TEAP were found for samples N7, N11 from north, A1, A8 from Azores Archipelago and $\mathrm{CC} 2, \mathrm{CC} 4$ from coast center, while samples A10, A11 from Azores Archipelago and all the south samples presented the lowest values. These electrochemical results follow the same profile of the other antioxidant methods used previously [27] for the evaluation of the antioxidant activity in the same samples, with the north, coast center and Azores samples showing the best scavenging effects, while south, CI2-CI4 from central interior and Madeira Island presented the lowest activities. Concerning the TEAP values presented by plant sources PM, PF 
Table 2 Electrochemical antioxidant power (EAP), obtained by differential pulse voltammetry in EtOH/ Britton-Robinson buffer/TBAP (78:20:2) solution at $\mathrm{pH} 7$

\begin{tabular}{|c|c|c|c|c|c|c|c|c|}
\hline \multirow[t]{2}{*}{ Sample } & \multicolumn{7}{|l|}{$\mathrm{EAP}^{\mathrm{a}}$} & \multirow[t]{2}{*}{ TEAP $^{\mathrm{b}}$} \\
\hline & $0.15 \mathrm{~V}$ & $0.25 \mathrm{~V}$ & $0.35 \mathrm{~V}$ & $0.55 \mathrm{~V}$ & $0.7 \mathrm{~V}$ & $0.85 \mathrm{~V}$ & $1.05 \mathrm{~V}$ & \\
\hline N1 & - & $24 \pm 2$ & - & $26 \pm 1$ & - & - & - & $49 \pm 2$ \\
\hline $\mathrm{N} 2$ & - & $15 \pm 1$ & - & $20 \pm 1$ & - & - & - & $35 \pm 2$ \\
\hline N3 & - & $18 \pm 2$ & - & $23 \pm 0$ & - & - & - & $42 \pm 2$ \\
\hline N4 & - & $11 \pm 2$ & - & $22 \pm 1$ & - & - & - & $33 \pm 3$ \\
\hline N5 & - & $16 \pm 2$ & - & $20 \pm 1$ & - & - & - & $36 \pm 2$ \\
\hline N6 & - & $12 \pm 0$ & - & $14 \pm 1$ & - & - & - & $26 \pm 1$ \\
\hline N7 & - & $16 \pm 1$ & $23 \pm 2$ & $21 \pm 3$ & - & - & - & $60 \pm 4$ \\
\hline N8 & - & $25 \pm 1$ & - & $24 \pm 1$ & - & - & $4 \pm 0$ & $53 \pm 2$ \\
\hline N9 & - & $17 \pm 0$ & - & $21 \pm 2$ & - & - & $4 \pm 0$ & $42 \pm 2$ \\
\hline N10 & - & $14 \pm 1$ & - & $15 \pm 1$ & - & - & $4 \pm 0$ & $33 \pm 1$ \\
\hline N11 & - & $11 \pm 2$ & $21 \pm 2$ & $18 \pm 1$ & - & $5 \pm 0$ & $5 \pm 0$ & $60 \pm 3$ \\
\hline N12 & - & $7 \pm 1$ & $11 \pm 1$ & $13 \pm 0$ & - & $4 \pm 0$ & $5 \pm 0$ & $40 \pm 2$ \\
\hline N13 & - & $5 \pm 0$ & $6 \pm 1$ & $10 \pm 0$ & - & $6 \pm 0$ & $9 \pm 0$ & $35 \pm 1$ \\
\hline N14 & - & $16 \pm 1$ & $13 \pm 1$ & $9 \pm 0$ & - & - & $6 \pm 0$ & $44 \pm 1$ \\
\hline CI1 & - & $10 \pm 1$ & $15 \pm 1$ & $8 \pm 1$ & $5 \pm 0$ & $5 \pm 0$ & $5 \pm 0$ & $47 \pm 2$ \\
\hline $\mathrm{CI} 3$ & - & $8 \pm 0$ & $8 \pm 1$ & $8 \pm 1$ & - & $7 \pm 1$ & $8 \pm 1$ & $39 \pm 1$ \\
\hline CI4 & - & $6 \pm 2$ & $8 \pm 3$ & $8 \pm 2$ & - & $5 \pm 0$ & $4 \pm 0$ & $30 \pm 4$ \\
\hline $\mathrm{CC} 1$ & - & $24 \pm 0$ & - & $25 \pm 0$ & - & - & - & $49 \pm 0$ \\
\hline $\mathrm{CC} 2$ & - & $12 \pm 3$ & - & $32 \pm 2$ & - & $20 \pm 0$ & - & $64 \pm 4$ \\
\hline $\mathrm{CC} 3$ & - & $19 \pm 1$ & - & $21 \pm 1$ & - & - & $5 \pm 0$ & $45 \pm 1$ \\
\hline $\mathrm{CC} 4$ & - & $27 \pm 3$ & & $23 \pm 2$ & - & - & $4 \pm 0$ & $53 \pm 4$ \\
\hline S1 & - & $9 \pm 1$ & - & $17 \pm 1$ & - & - & $3 \pm 0$ & $29 \pm 2$ \\
\hline S2 & - & $5 \pm 0$ & - & $6 \pm 0$ & - & $10 \pm 1$ & $7 \pm 0$ & $28 \pm 1$ \\
\hline S3 & - & $7 \pm 1$ & - & $7 \pm 0$ & - & $7 \pm 0$ & $5 \pm 0$ & $26 \pm 1$ \\
\hline S4 & - & $4 \pm 1$ & - & $6 \pm 1$ & - & $5 \pm 0$ & $5 \pm 0$ & $20 \pm 1$ \\
\hline A1 & $21 \pm 1$ & $24 \pm 2$ & - & $25 \pm 1$ & - & - & $4 \pm 0$ & $73 \pm 3$ \\
\hline $\mathrm{A} 2$ & - & $17 \pm 0$ & - & $33 \pm 1$ & - & - & - & $50 \pm 1$ \\
\hline A 3 & - & $13 \pm 1$ & - & $26 \pm 1$ & - & - & - & $39 \pm 1$ \\
\hline A4 & $9 \pm 0$ & $14 \pm 0$ & - & $26 \pm 0$ & - & - & - & $49 \pm 1$ \\
\hline A5 & - & $18 \pm 2$ & - & $29 \pm 1$ & - & - & - & $47 \pm 2$ \\
\hline A6 & - & $19 \pm 2$ & - & $27 \pm 1$ & - & - & - & $46 \pm 2$ \\
\hline A7 & $12 \pm 3$ & - & - & $30 \pm 4$ & - & - & - & $41 \pm 5$ \\
\hline A8 & $11 \pm 0$ & $17 \pm 0$ & - & $24 \pm 1$ & - & $11 \pm 1$ & - & $63 \pm 1$ \\
\hline A9 & - & $11 \pm 0$ & - & $18 \pm 1$ & - & - & - & $30 \pm 1$ \\
\hline A 10 & $11 \pm 2$ & - & - & - & - & - & - & $11 \pm 2$ \\
\hline A11 & - & $9 \pm 1$ & - & - & - & - & - & $9 \pm 1$ \\
\hline M1 & - & $8 \pm 1$ & $4 \pm 0$ & - & - & $6 \pm 0$ & - & $17 \pm 1$ \\
\hline M2 & - & $19 \pm 4$ & $14 \pm 2$ & - & $22 \pm 0$ & - & - & $56 \pm 4$ \\
\hline M3 & - & - & - & $15 \pm 4$ & - & $13 \pm 2$ & - & $28 \pm 4$ \\
\hline PM & - & $15 \pm 0$ & - & $15 \pm 1$ & - & - & - & $30 \pm 1$ \\
\hline $\mathrm{PF}$ & - & $6 \pm 0$ & - & $9 \pm 0$ & - & - & - & $15 \pm 0$ \\
\hline $\mathrm{C}$ & - & $12 \pm 0$ & $18 \pm 1$ & - & - & $5 \pm 0$ & - & $35 \pm 1$ \\
\hline
\end{tabular}

a Electrochemical antioxidant power

b Total electrochemical antioxidant power, at $\mathrm{pH}$ 7, expressed in $\mathrm{mgg}^{-1}$ of caffeic acid: galangin: pinocembrin $(1: 1: 1)$ at the different potentials presented by the propolis and plant sources extracts (mean $\pm \mathrm{SD}$; $n=3)$ 
and $\mathrm{C}$, the antioxidant activity is significantly lower than the propolis samples, with values of 30,15 and $35 \mathrm{mgg}^{-1}$, respectively. This could be explained by the fact that propolis itself is a product of a selection and concentration of different resins undertaken by bees, and so its richness in electroactive species is higher than each plant source itself.

\section{Conclusions}

Electrochemical methods such as cyclic voltammetry and differential pulse voltammetry were applied as a fast an easy tool for the evaluation of the redox profile and the quantification of the total antioxidant capacity in Portuguese propolis from different origins. Both techniques showed the existence of several oxidation processes which vary with the origin of the resin, but reveal common oxidation potentials within the majority of samples of a region suggesting an analogous chemical composition in respect of electroactive species. The electrochemical profile of Cistus ladanifer resembles that of propolis samples N11-N13 from the north, CI1-CI4 from central interior, M1 from Madeira Island and samples S2-S4 from south due to the presence of both oxidation processes at 0.85 and $1.05 \mathrm{~V}$, confirming the botanical discrimination already observed for these samples with other analytical tools. Considering this pattern, the electrochemical profile can be used as a quick method to identify Cistus Portuguese propolis. With the quantification of the total electroactive antioxidant power, it was possible to attribute the higher antioxidant activity to propolis from coast center, followed by north $\sim$ Azores Archipelago $>$ central interior $>$ Madeira Island $>$ south.

Acknowledgments Soraia I. Falcão thanks Foundation for Science and Technology (FCT) for the PhD grant SFRH/BD/44855/2008, financially supported by POPH-QREN and FSE. Thanks also to FCT for financial support provided to CIMO (PEst-OE/AGR/ UI0690/2011). Thanks to National Federation of Portuguese Beekeepers for propolis samples.

\section{Compliance with ethical standards}

Conflict of interest The authors declare that they have no conflict of interest.

Compliance with ethics requirements This article does not contain any studies with human or animal subjects.

\section{References}

1. Rice-Evans CA, Parker L (1998) Flavonoids in health and disease. Marcell Dekker, New York

2. Snapos G, Wrolstad R (1992) Phenolics of apple, pear, and white grape juices and their changes with processing and storage. A review. J Agric Food Chem 40:1478-1487
3. Segura M, Novak I, Jakobek L (2011) Determination of polyphenols content and antioxidant activity of some red wines by differential pulse voltammetry, HPLC and spectrophotometric methods. Food Chem 124:1208-1216

4. Gómez-Caravac AM, Gómez-Romero M, Arráez-Román D, Segura-Carretero A, Fernández-Gutiérrez A (2006) Advances in the analysis of phenolic compounds in products derived from bees. $\mathrm{J}$ Pharmaceut Biomed Anal 46:1220-1234

5. Bankova VS, De Castro SL, Marcucci MC (2000) Propolis: recent advances in chemistry and plant origin. Apidologie 3:13-15

6. Salatino A, Fernandes-Silva CC, Righi AA, Salatino MFL (2011) Propolis research and the chemistry of plant products. Nat Prod Rep 28:925-936

7. Falcão SI, Vale N, Gomes P, Domingues MRM, Freire C, Cardoso SM, Vilas-Boas M (2013) Phenolic profiling of Portuguese propolis by LC-MS spectrometry: uncommon propolis rich with flavonoid glycosides. Phytochem Anal 24(4):309-318

8. Sforcin JM, Bankova V (2011) Propolis: is there a potential for the development of new drugs? J Ethnopharmacol 133:253-260

9. Kumazawa S, Hamasaka T, Nakayawa T (2004) Antioxidant activity of propolis of various geographic origins. Food Chem 84:329-339

10. Jasprica I, Bojic M, Mornar A, Basic E, Bucan K, Medic-Saric M (2007) Evaluation of antioxidative activity of croatian propolis samples using DPPH and ABTS + stable free radical assays. Molecules 12:1006-1021

11. Blasco AJ, Crevillén AG, González MC, Escarpa A (2007) Direct electrochemical sensing and detection of natural antioxidants and antioxidant capacity in vitro systems. Electroanal. 22:2275-2286

12. Huang D, Ou B, Prior RL (2005) The chemistry behind antioxidant capacity assays. J Agric Food Chem 53:1841-1856

13. Mannino S, Brenna O, Buratti S, Cosio MS (1998) A new method for the evaluation of the 'antioxidant power' of wines. Electroanal 10:908-912

14. Magalhães LM, Segundo MA, Reis S, Lima JLFC (2008) Methodological aspects about in vitro evaluation of antioxidant properties. Anal Chim Acta 613:1-19

15. Buratti S, Benedetti S, Cosio MS (2007) Evaluation of the antioxidant power of honey, propolis and royal jelly by amperometric flow injection analysis. Talanta 71:1387-1392

16. Laskar RA, Sk I, Roy N, Begum NA (2010) Antioxidant activity of Indian propolis and its chemical constituents. Food Chem 122:233-237

17. Potkonjak NI, Veselinović DS, Novaković MM, Gorjanović SŽ, Pezo LL, Sužnjević DŽ (2012) Antioxidant activity of propolis extracts from Serbia: a polarographic approach. Food Chem Toxicol 50:3614-3618

18. Rapta P, Mišík V, Staško A, Vrábel I (1995) Redox intermediates of flavonoids and caffeic acid esters from propolis: an EPR spectroscopy and cyclic voltammetry study. Free Radic Biol Med 18:901-908

19. Barros L, Falcão S, Baptista P, Freire C, Vilas-Boas M, Ferreira ICFR (2008) Antioxidant activity of Agaricus sp. Mushrooms by Chemical, biochemical and electrochemical assays. Food Chem 111:61-66

20. Barros L, Cabrita L, Vilas-Boas M, Carvalho AM, Ferreira ICFR (2011) Chemical, biochemical and electrochemical assays to evaluate phytochemicals and antioxidant activity of wild plants. Food Chem 127:1600-1608

21. Calhelha R, Peixoto D, Vilas-Boas M, Queiroz MJRP, Ferreira ICFR (2013) Antioxidant activity of aminodiarylamines in the thieno[3,2-b]pyridine series: radical scavenging activity, lipid peroxidation inhibition and redox profile. J Enzym Inhib Med Chem 29:311-316 
22. Martos I, Cossentini M, Ferreres F, Tomás-Barberán FA (1997) Flavonoid composition of Tunisian honeys and propolis. J Agric Food Chem 45:2824-2829

23. Jovanovic SV, Steenken S, Tosic M, Marjanovic B, Simic M (1994) Flavonoids as antioxidants. J Am Chem Soc 116:4846

24. Blasco AJ, González MC, Escarpa A (2004) Electrochemical approach for discriminating and measuring predominant flavonoids and phenolic acids using differential pulse voltammetry: towards an electrochemical index of natural antioxidants. Anal Chim Acta 511:71-81
25. Arribas AS, Martínez-Fernandes M, Chicharro M (2012) The role of electroanalytical techniques in analysis of polyphenols in wine. Trend Anal Chem 34:78-96

26. Brett CMA, Brett AMO (1993) Electrochemistry-principles methods and applications. Oxford University Press, Oxford

27. Falcão SI, Freire C, Vilas-Boas M (2013) A proposal for quality standards and antioxidant activity of Portuguese propolis. J Am Oil Chem Soc 90:1729-1741

28. Falcão SI, Tomás A, Vale N, Gomes P, Freire C, Vilas-Boas M (2013) Phenolic quantification and botanical origin of Portuguese propolis. Ind Crop Prod 49:805-812 